\title{
Awareness regarding the strategies to bring down the pandemic among general population: a questionnaire based study
}

\author{
Varsha Christy Rani Balaji*, Nithya R.
}

Department of Obstetrics and Gynecology, Sree Balaji Medical College and Hospital Chrompet, Chennai, Tamil Nadu, India

Received: 07 January 2021

Accepted: 09 February 2021

*Correspondence:

Dr. Varsha Christy Rani Balaji,

E-mail: vershabalaji@gmail.com

Copyright: (c) the author(s), publisher and licensee Medip Academy. This is an open-access article distributed under the terms of the Creative Commons Attribution Non-Commercial License, which permits unrestricted non-commercial use, distribution, and reproduction in any medium, provided the original work is properly cited.

\begin{abstract}
Background: The behavior of the general public will likely have an important effect on how the coronavirus disease 2019 (COVID-19) epidemic spreads, especially when the first wave is diminishing. The aim of the study is to determine the awareness regarding the strategies to bring down the pandemic among the general population of Tamil Nadu in South India.

Methods: A cross-sectional online survey conducted in October 2020. A sample of consenting 500 adult, healthy caregivers of patients attending a tertiary medical institute who were representative of the general population formed the study group. They filled a pre-formed questionnaire. All data entry and analysis were performed using SPSS (version 24). Descriptive statistics and chi-square test were performed between the various sociodemographic factors of age, gender, occupation, and education, and COVID-19 exposure in the family. $\mathrm{P} \leq 0.05$ was taken to be significant. Results: It was observed that age had a significant influence on the wearing of masks and the use of hand sanitizer. Gender, age and education are the important determinants for use of hand sanitizer, wearing a mask, practicing social distancing, avoid traveling to the infected areas as well as washing hands often and always using a facemask to prevent COVID-19 were statistically significant.

Conclusions: The dynamics of the impact of the awareness campaign and public participation are understood by the results. There is a need for a training program with respect to locale-specific scenario targeted to a specific cluster of population emplaning upon their respective lifestyle, to improve the knowledge and compliance about risk and preventions.
\end{abstract}

Keywords: Awareness, COVID-19, Education, Handwash, Impact, Mask, Sanitizer

\section{INTRODUCTION}

The novel Corona Viral Disease of 2019 (COVID-19) was declared as a pandemic in March 2020 and spread at an unimaginable rate causing a heavy burden on worldwide healthcare systems, forcing almost most of the countries to apply quarantine rules. COVID-19 traits such as high transmission rates, long incubation period - 7 to 14 days - and global spread, infecting hundreds of thousands of people, indicated that the virus needed to be tackled with careful design and planning. Initial reports from China revealed that the primary symptoms of Covid-19 to be fever, dry coughs, and shortness of breath. As there was a lack of appropriate vaccine or cure for treating and managing these patients, the best way in current situation is paying special attention to disease prevention and breaking the transmission chain of the virus. There was no clear cut evidence on route of infection and portal of entry of virus into humans. Hence at the initial phases of infection, experts believed that COVID-19 prevention would requires stringent personal and public hygiene, especially related to respiratory and 
contact. These includes washing hands with soap or alcohol "medical grade sanitizers" for at least twenty seconds, reducing interactions with other people to the minimum, quarantining people who have come into contact with anyone infected as well as advices to wear mask when interacting with people and maintaining social distancing of 6 feet. Actions like these need a social awareness from both the authorities and the population at large, in order to handle the COVID-19 situation quickly and safely. These advisories were strictly implemented with fines and other legal monitoring guidelines. ${ }^{1,2}$

Since late May 2020, India began "COVID-19" unlocking procedures. Tamil Nadu reported its first COVID-19 case on 5th March 2020 and the earliest clusters of locally acquired cases emerged in March in Chennai and surrounding coastal districts of eastern Tamil Nadu. Of all districts, Chennai ultimately experienced the highest cumulative incidence of COVID19 , totaling 102,199 cases (204.6 per 10,000 population) by 1 August 2020. The overall secondary attack rate (or risk of transmission from an index case to an exposed contact) in Tamil Nadu was $10.7 \%$ for high-risk contacts, who had close social contact or direct physical contact with index cases without protective measures, and $4.7 \%$ for low-risk contacts, who were in the proximity of index cases but did not meet these criteria for high-risk exposure. There has been data available about exposure settings for 18,485 contacts of 1343 index cases. This revealed considerable differences in transmission risk associated with differing types of interaction. Secondary attack rate estimates ranged from $1.2 \%$ in health care settings to $2.6 \%$ in the community and $9.0 \%$ in the household. ${ }^{3}$ Given these figures in Tamil Nadu, a pilot attempt was made assess the Chennai, India general public's awareness on COVID-19 mitigation strategies and observe the effect of common socio-demographic factors on such awareness. Such as study would help to frame future studies and design loco-regional specific health policies.

\section{METHODS}

This cross-sectional survey was conducted from October 1st to October 31st 2020, to assess the Chennai, India general public's awareness on COVID-19 mitigation strategies. As the disease process is dynamic at the phase of study in Chennai, India, a convenient sample of 500 was done to get the initial screening estimate to plan future studies. The survey questionnaire included both prompted direct questions as well as unprompted open ended questions that allowed for multiple responses. The Coronavirus related questions on infection spread were aggregated in the questionnaire from sources of information, knowledge about Coronavirus spread, precautions and widely advised government prevention practices. Before the final survey was undertaken, changes were made as required to enable better understanding of the questions by the participants, and the arrangement of the questions was looked into to ensure its efficiency. English and vernacular translation (after back translation verified independently) was circulated.

\section{Data collection}

While maintaining social distance, the survey was done among the public attending the institution. People who were attending the Outpatient ward with no disease (attenders of patients) were asked to participate in the survey and directed to complete the questionnaire. The survey started with a sentence that "completing the questionnaire by the participants is considered as voluntary participation". After confirmation that participants understood this, people were guided to complete the self-report questionnaire. On average, questionnaires took approximately 5 minutes to complete.

\section{Data analysis}

All data entry and analysis was performed using statistical package for social services (version 24). Descriptive statistics and chi-square test were performed between the various sociodemographic factors of age, gender, occupation, education and COVID-19 exposure in the family. $\mathrm{P} \leq 0.05$ was taken to be significant.

\section{RESULTS}

In all 500 valid, completed, consenting participants data were collected for the purpose of this study. Participant's awareness of the preventive measure towards COVID-19. The socio-demographic parameter's frequency and distribution are depicted in Table 1. The overall report of the study population $(n=500)$ is also shown in Table-1. The Table 2 shows the effect of age on the various study parameters. Of that, it was observed that age had a significant influence on the wearing of mask, use of hand sanitizer and isolation to be significantly varying between different age group $(\mathrm{P}=0.000)$. Table 3 shows the study population $(n=500)$ classified by the gender shows that using hand sanitizer and wearing mask often and avoid travel to infected area often shows a significant result. Table 4 classifies according to the occupation and shows that using hand sanitizer, wearing mask often, practicing social distancing, washing hands often and avoiding travel to infected area and following these measures are effective and statistically significant. Table 5 classifies the familial impact of COVID-19 exposure in the study population $(n=500)$ the measure such as practicing social distancing, avoiding travel to infected area are statistically significant. The Table 6 classifies according to the level of education and shows that isolating people from infected area and using hand sanitizer, wearing mask, practicing social distancing, avoid travelling to infected area and washing hands often and always using face mask to prevent COVID-19 are statistically significant (Table 1-6). 
Table 1: Socio-demographics and consolidated replies by the participants $(n=500)$.

\begin{tabular}{|c|c|c|c|}
\hline Socio-demographics & & Frequency & Percent \\
\hline \multirow{5}{*}{ Age group } & $\begin{array}{l}25 \text { years and } \\
\text { below }\end{array}$ & 59 & 11.8 \\
\hline & 26 to 35 years & 107 & 21.4 \\
\hline & 36 to 45 years & 116 & 23.2 \\
\hline & 46 to 55 years & 123 & 24.6 \\
\hline & Above 56 years & 95 & 19 \\
\hline \multirow{2}{*}{ Gender } & Female & 266 & 53.2 \\
\hline & Male & 234 & 46.8 \\
\hline \multirow[t]{5}{*}{ Academic Qualification } & No Formal & 90 & 18 \\
\hline & Primary & 56 & 11.2 \\
\hline & Middle & 86 & 17.2 \\
\hline & Higher Secondary & 148 & 29.6 \\
\hline & Collegiate & 120 & 24 \\
\hline \multirow{5}{*}{ Nature of employment } & Managerial & 168 & 33.6 \\
\hline & Others & 34 & 6.8 \\
\hline & Professional & 51 & 10.2 \\
\hline & Skilled & 211 & 42.2 \\
\hline & Unskilled & 36 & 7.2 \\
\hline Are you aware of COVID-19? & Yes & 500 & 100 \\
\hline \multirow{2}{*}{ Any family member suffered from COVID-19 } & No & 434 & 86.8 \\
\hline & Yes & 66 & 13.2 \\
\hline Avoid social distancing to prevent COVID-19 spread & Yes & 500 & 100 \\
\hline Wear mask to prevent spread of COVID-19 & Yes & 500 & 100 \\
\hline \multirow{2}{*}{ Use hand sanitizer to prevent spread of COVID-19 } & No & 16 & 3.2 \\
\hline & Yes & 484 & 96.8 \\
\hline \multirow{2}{*}{$\begin{array}{l}\text { Isolating people from infected area would prevent } \\
\text { COVID-19 spread }\end{array}$} & No & 5 & 1 \\
\hline & Yes & 495 & 99 \\
\hline \multirow{2}{*}{ How often do you wear mask? } & Always & 479 & 95.8 \\
\hline & Sometimes & 21 & 4.2 \\
\hline \multirow{3}{*}{ How often do you practice social distancing } & Always & 443 & 88.6 \\
\hline & Rarely & 10 & 2 \\
\hline & Sometimes & 47 & 9.4 \\
\hline \multirow{3}{*}{ How often do you avoid travel to infected area } & Always & 387 & 77.4 \\
\hline & Rarely & 12 & 2.4 \\
\hline & Sometimes & 101 & 20.2 \\
\hline \multirow{3}{*}{ How often do you wash hands } & Always & 419 & 83.8 \\
\hline & Rarely & 5 & 1 \\
\hline & Sometimes & 76 & 15.2 \\
\hline \multirow{2}{*}{ Do you think chemical disinfectant can prevent COVID-19 } & No & 12 & 2.4 \\
\hline & Yes & 488 & 97.6 \\
\hline \multirow{2}{*}{$\begin{array}{l}\text { Do you think COVID-19 can be disinfected by natural } \\
\text { substances like turmeric etc., }\end{array}$} & No & 6 & 1.2 \\
\hline & Yes & 494 & 98.8 \\
\hline \multirow{3}{*}{$\begin{array}{l}\text { Do you think that to prevent contracting and spreading of } \\
\text { COVID-19 avoiding unnecessary vacations, consuming } \\
\text { outdoor food, avoiding going out of home, avoiding hand } \\
\text { shaking hugging and kissing, avoiding public } \\
\text { transportation, frequently washing hands will be more } \\
\text { effective? }\end{array}$} & No & 3 & 0.6 \\
\hline & No Opinion & 103 & 20.6 \\
\hline & Yes & 394 & 78.8 \\
\hline \multirow[b]{2}{*}{$\begin{array}{l}\text { When do you use face mask to prevent COVID-19 } \\
\text { contracting }\end{array}$} & Always & 362 & 72.4 \\
\hline & $\begin{array}{l}\text { Only in public } \\
\text { and crowded } \\
\text { places }\end{array}$ & 138 & 27.6 \\
\hline
\end{tabular}


Balaji VCR et al. Int J Reprod Contracept Obstet Gynecol. 2021 Mar;10(3):1077-1088

Table 2: Chi-square test result of various study parameters by the age group.

\begin{tabular}{|c|c|c|c|c|c|c|c|c|c|c|c|c|}
\hline \multirow[t]{2}{*}{$\begin{array}{l}\text { Various study } \\
\text { parameters }\end{array}$} & & \multicolumn{2}{|c|}{$\begin{array}{l}25 \text { years and } \\
\text { below }\end{array}$} & \multicolumn{2}{|c|}{26 - 35 years } & \multicolumn{2}{|c|}{$36-45$ years } & \multicolumn{2}{|c|}{46 to 55 years } & \multicolumn{2}{|c|}{$\begin{array}{l}56 \text { years and } \\
\text { above }\end{array}$} & \multirow[t]{2}{*}{ P value } \\
\hline & & Count & $\begin{array}{l}\text { Row } \\
\text { N \% }\end{array}$ & Count & $\begin{array}{l}\text { Row } \\
\text { N \% }\end{array}$ & Count & $\begin{array}{l}\text { Row } \\
\text { N \% }\end{array}$ & Count & $\begin{array}{l}\text { Row } \\
\text { N \% }\end{array}$ & Count & $\begin{array}{l}\text { Row N } \\
\%\end{array}$ & \\
\hline $\begin{array}{l}\text { Avoid social } \\
\text { distancing to prevent } \\
\text { COVID-19 spread }\end{array}$ & Yes & 59 & 11.80 & 107.0 & 21.40 & 116.0 & 23.20 & 123.0 & 24.60 & 59 & 11.80 & $\begin{array}{l}\text { Not } \\
\text { Computabl } \\
\text { e }\end{array}$ \\
\hline $\begin{array}{l}\text { Wear mask to prevent } \\
\text { spread of COVID-19 }\end{array}$ & Yes & 59 & 11.80 & 107.00 & 21.40 & 116.00 & 23.20 & 123.00 & 24.60 & 59 & 11.80 & $\begin{array}{l}\text { Not } \\
\text { Computabl } \\
\text { e }\end{array}$ \\
\hline \multirow{2}{*}{$\begin{array}{l}\text { Use hand sanitizer to } \\
\text { prevent spread of } \\
\text { COVID-19 }\end{array}$} & No & 0 & 0.00 & 0.00 & 0.00 & 5.00 & 31.25 & 1.00 & 6.25 & 0 & 0.00 & \multirow{2}{*}{0.000} \\
\hline & Yes & 59 & 12.19 & 107.00 & 22.11 & 111.00 & 22.93 & 122.00 & 25.21 & 59 & 12.19 & \\
\hline \multirow{2}{*}{$\begin{array}{l}\text { Isolating people from } \\
\text { infected area would } \\
\text { prevent COVID-19 } \\
\text { spread }\end{array}$} & No & 0 & 0.00 & 0.00 & 0.00 & 0.00 & 0.00 & 5.00 & 100.00 & 0 & 0.00 & \multirow{2}{*}{0.004} \\
\hline & Yes & 59 & 11.92 & 107.00 & 21.62 & 116.00 & 23.43 & 118.00 & 23.84 & 59 & 11.92 & \\
\hline \multirow{2}{*}{$\begin{array}{l}\text { How often do you } \\
\text { wear mask? }\end{array}$} & Always & 54 & 11.27 & 107.00 & 22.34 & 110.00 & 22.96 & 118.00 & 24.63 & 54 & 11.27 & \multirow{2}{*}{0.095} \\
\hline & sometimes & 5 & 23.81 & 0.00 & 0.00 & 6.00 & 28.57 & 5.00 & 23.81 & 5 & 23.81 & \\
\hline \multirow{3}{*}{$\begin{array}{l}\text { How often do you } \\
\text { practice social } \\
\text { distancing }\end{array}$} & Always & 49 & 11.06 & 97.00 & 21.90 & 104.00 & 23.48 & 103.00 & 23.25 & 49 & 11.06 & \multirow{3}{*}{0.000} \\
\hline & Rarely & 5 & 50.00 & 5.00 & 50.00 & 0.00 & 0.00 & 0.00 & 0.00 & 5 & 50.00 & \\
\hline & Sometimes & 5 & 10.64 & 5.00 & 10.64 & 12.00 & 25.53 & 20.00 & 42.55 & 5 & 10.64 & \\
\hline \multirow{3}{*}{$\begin{array}{l}\text { How often do you } \\
\text { avoid travel to } \\
\text { infected area }\end{array}$} & Always & 47 & 12.14 & 81.00 & 20.93 & 81.00 & 20.93 & 98.00 & 25.32 & 47 & 12.14 & \multirow{3}{*}{0.000} \\
\hline & Rarely & 0 & 0.00 & 0.00 & 0.00 & 12.00 & 100.00 & 0.00 & 0.00 & 0 & 0.00 & \\
\hline & Sometimes & 12 & 11.88 & 26.00 & 25.74 & 23.00 & 22.77 & 25.00 & 24.75 & 12 & 11.88 & \\
\hline \multirow{3}{*}{$\begin{array}{l}\text { How often do you } \\
\text { wash hands }\end{array}$} & Always & 54 & 12.89 & 91.00 & 21.72 & 96.00 & 22.91 & 108.00 & 25.78 & 54 & 12.89 & \multirow{3}{*}{0.000} \\
\hline & rarely & 0 & 0.00 & 0.00 & 0.00 & 0.00 & 0.00 & 5.00 & 100.00 & 0 & 0.00 & \\
\hline & Sometimes & 5 & 6.58 & 16.00 & 21.05 & 20.00 & 26.32 & 10.00 & 13.16 & 5 & 6.58 & \\
\hline \multirow{2}{*}{$\begin{array}{l}\text { Do you think } \\
\text { chemical disinfectant } \\
\text { can prevent COVID- } \\
19\end{array}$} & No & 1 & 8.33 & 3.00 & 25.00 & 2.00 & 16.67 & 1.00 & 8.33 & 1 & 8.33 & \multirow[b]{2}{*}{0.280} \\
\hline & yes & 58 & 11.89 & 104.00 & 21.31 & 114.00 & 23.36 & 122.00 & 25.00 & 58 & 11.89 & \\
\hline \multirow{2}{*}{$\begin{array}{l}\text { Do you think COVID- } \\
19 \text { can be disinfected } \\
\text { by natural substances } \\
\text { like turmeric etc., }\end{array}$} & No & 1 & 16.67 & 1.00 & 16.67 & 1.00 & 16.67 & 3.00 & 50.00 & 1 & 16.67 & \multirow[b]{2}{*}{0.551} \\
\hline & Yes & 58 & 11.7 & 106 & 21.5 & 115 & 23.3 & 120 & 24.3 & 95 & 19.2 & \\
\hline \multirow{2}{*}{$\begin{array}{l}\text { Do you think that to } \\
\text { prevent contracting }\end{array}$} & No & 1 & 33.33 & 1.00 & 33.33 & 0.00 & 0.00 & 0.00 & 0.00 & 1 & 33.33 & \multirow{2}{*}{0.173} \\
\hline & No Opinion & 10 & 9.71 & 25.00 & 24.27 & 26.00 & 25.24 & 16.00 & 15.53 & 10 & 9.71 & \\
\hline
\end{tabular}


Balaji VCR et al. Int J Reprod Contracept Obstet Gynecol. 2021 Mar;10(3):1077-1088

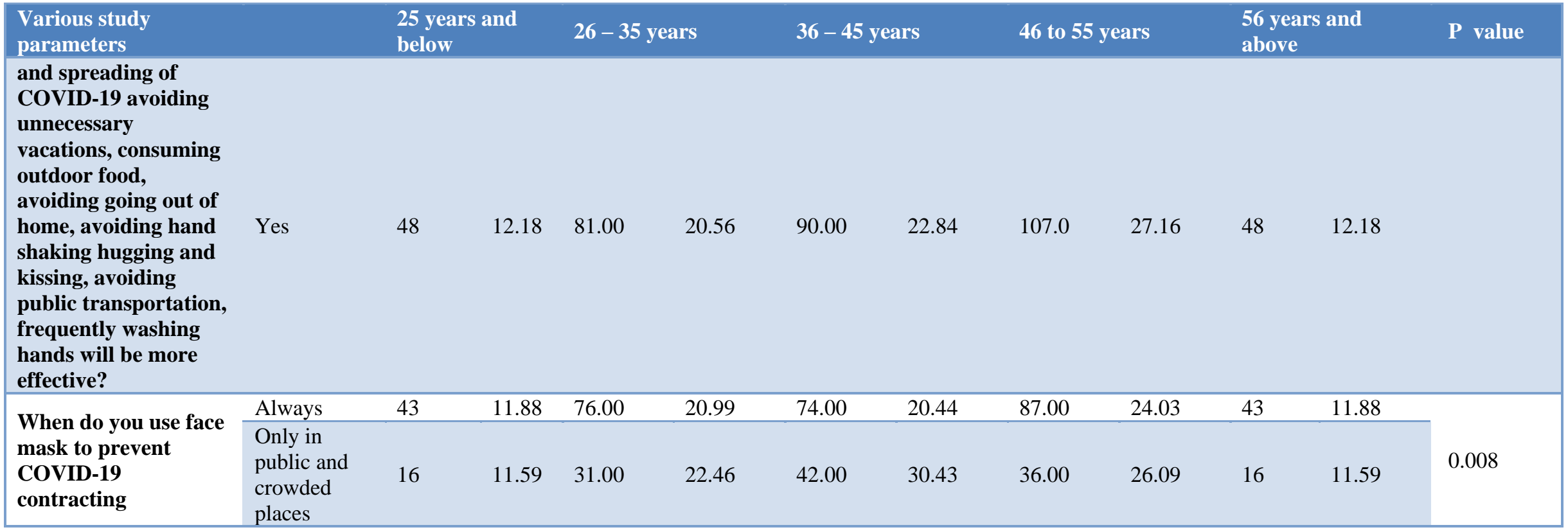

Table 3: Various study parameters by the gender $(n=500)$.

\begin{tabular}{|c|c|c|c|c|c|c|}
\hline \multirow{3}{*}{ Study parameters } & & \multicolumn{4}{|l|}{ Sex } & \multirow{3}{*}{ P value } \\
\hline & & \multicolumn{2}{|l|}{ Female } & \multicolumn{2}{|l|}{ Male } & \\
\hline & & Count & Row N \% & Count & Row $\mathbf{N} \%$ & \\
\hline $\begin{array}{l}\text { Avoid social distancing to prevent } \\
\text { COVID-19 spread }\end{array}$ & Yes & 266 & 53.20 & 234 & 46.80 & Not computable \\
\hline $\begin{array}{l}\text { Wear mask to prevent spread of COVID- } \\
19\end{array}$ & Yes & 266 & 53.20 & 234 & 46.80 & Not computable \\
\hline \multirow{2}{*}{$\begin{array}{l}\text { Use hand sanitizer to prevent spread of } \\
\text { COVID-19 }\end{array}$} & No & 15 & 93.80 & 1 & 6.20 & \multirow{2}{*}{0.001} \\
\hline & Yes & 251 & 51.90 & 233 & 48.10 & \\
\hline \multirow{2}{*}{$\begin{array}{l}\text { Isolating people from infected area would } \\
\text { prevent COVID-19 spread }\end{array}$} & No & 1 & 20.00 & 4 & 80.00 & \multirow{2}{*}{0.149} \\
\hline & Yes & 265 & 53.50 & 230 & 46.50 & \\
\hline \multirow{2}{*}{ How often do you wear mask? } & Always & 261 & 54.50 & 218 & 45.50 & \multirow{2}{*}{0.005} \\
\hline & Sometimes & 5 & 23.80 & 16 & 76.20 & \\
\hline \multirow{3}{*}{$\begin{array}{l}\text { How often do you practice social } \\
\text { distancing }\end{array}$} & Always & 238 & 53.70 & 205 & 46.30 & \multirow{3}{*}{0.805} \\
\hline & Rarely & 5 & 50.00 & 5 & 50.00 & \\
\hline & Sometimes & 23 & 48.90 & 24 & 51.10 & \\
\hline \multirow{2}{*}{$\begin{array}{l}\text { How often do you avoid travel to infected } \\
\text { area }\end{array}$} & Always & 213 & 55.00 & 174 & 45.00 & \multirow[t]{2}{*}{0.000} \\
\hline & Rarely & 12 & 100.00 & 0 & 0.00 & \\
\hline
\end{tabular}

International Journal of Reproduction, Contraception, Obstetrics and Gynecology 


\begin{tabular}{|c|c|c|c|c|c|c|}
\hline \multirow{3}{*}{ Study parameters } & \multicolumn{5}{|c|}{ Sex } & \multirow{3}{*}{$P$ value } \\
\hline & & \multicolumn{2}{|l|}{ Female } & \multicolumn{2}{|l|}{ Male } & \\
\hline & & Count & Row N \% & Count & Row $\mathbf{N} \%$ & \\
\hline & Sometimes & 41 & 40.60 & 60 & 59.40 & \\
\hline \multirow{3}{*}{ How often do you wash hands } & Always & 225 & 53.70 & 194 & 46.30 & \multirow{3}{*}{0.057} \\
\hline & rarely & 0 & 0.00 & 5 & 100.00 & \\
\hline & Sometimes & 41 & 53.90 & 35 & 46.10 & \\
\hline \multirow{2}{*}{$\begin{array}{l}\text { Do you think chemical disinfectant can } \\
\text { prevent COVID-19 }\end{array}$} & No & 9 & 75.00 & 3 & 25.00 & \multirow{2}{*}{0.106} \\
\hline & yes & 257 & 52.70 & 231 & 47.30 & \\
\hline \multirow{2}{*}{$\begin{array}{l}\text { Do you think COVID-19 can be } \\
\text { disinfected by natural substances like } \\
\text { turmeric etc., }\end{array}$} & No & 2 & 33.30 & 4 & 66.70 & \multirow[b]{2}{*}{0.285} \\
\hline & Yes & 264 & 53.40 & 230 & 46.60 & \\
\hline \multirow{3}{*}{$\begin{array}{l}\text { Do you think that to prevent contracting } \\
\text { and spreading of COVID-19 avoiding } \\
\text { unnecessary vacations, consuming } \\
\text { outdoor food, avoiding going out of home, } \\
\text { avoiding hand shaking hugging and } \\
\text { kissing, avoiding public transportation, } \\
\text { frequently washing hands will be more } \\
\text { effective? }\end{array}$} & No & 0 & 0.00 & 3 & 100.00 & \multirow[b]{3}{*}{0.124} \\
\hline & No Opinion & 59 & 57.30 & 44 & 42.70 & \\
\hline & Yes & 207 & 52.50 & 187 & 47.50 & \\
\hline \multirow[b]{2}{*}{$\begin{array}{l}\text { When do you use face mask to prevent } \\
\text { COVID-19 contracting }\end{array}$} & Always & 199 & 55.00 & 163 & 45.00 & \multirow[b]{2}{*}{0.118} \\
\hline & $\begin{array}{l}\text { Only in public } \\
\text { and crowded } \\
\text { places }\end{array}$ & 67 & 48.60 & 71 & 51.40 & \\
\hline
\end{tabular}

Table 4: Study population (n=500) compared by nature of occupation $(n=500)$.

\begin{tabular}{|c|c|c|c|c|c|c|c|c|c|c|c|c|}
\hline & & \multicolumn{2}{|c|}{ Managerial } & \multicolumn{2}{|l|}{ Others } & \multicolumn{2}{|c|}{ Professional } & \multicolumn{2}{|l|}{ Skilled } & \multicolumn{2}{|l|}{ Unskilled } & \multirow[t]{2}{*}{$P$ value } \\
\hline & & Count & $\begin{array}{l}\text { Row N } \\
\%\end{array}$ & Count & $\begin{array}{l}\text { Row N } \\
\%\end{array}$ & Count & $\begin{array}{l}\text { Row N } \\
\%\end{array}$ & Count & $\begin{array}{l}\text { Row N } \\
\%\end{array}$ & Count & $\begin{array}{l}\text { Row N } \\
\%\end{array}$ & \\
\hline $\begin{array}{l}\text { Avoid social distancing } \\
\text { to prevent COVID-19 } \\
\text { spread }\end{array}$ & Yes & 168 & 33.6 & 34 & 6.8 & 51 & 10.2 & 211 & 42.2 & 36 & 7.2 & Not computable \\
\hline $\begin{array}{l}\text { Wear mask to prevent } \\
\text { spread of COVID-19 }\end{array}$ & Yes & 168 & 33.6 & 34 & 6.8 & 51 & 10.2 & 211 & 42.2 & 36 & 7.2 & Not computable \\
\hline \multirow{2}{*}{$\begin{array}{l}\text { Use hand sanitizer to } \\
\text { prevent spread of } \\
\text { COVID-19 }\end{array}$} & No & 1 & 6.2 & 0 & .0 & 0 & .0 & 10 & 62.0 & 5 & 31.2 & \multirow{2}{*}{0.000} \\
\hline & Yes & 167 & 34.5 & 34 & 7.0 & 51 & 10.5 & 201 & 41.5 & 31 & 6.4 & \\
\hline \multirow{2}{*}{$\begin{array}{l}\text { Isolating people from } \\
\text { infected area would } \\
\text { prevent COVID-19 }\end{array}$} & No & 4 & 80.0 & 0 & .0 & 0 & .0 & 1 & 20.0 & 0 & 0 & \multirow[t]{2}{*}{0.282} \\
\hline & Yes & 164 & 33.1 & 34 & 6.9 & 51 & 10.3 & 210 & 42.4 & 36 & 7.3 & \\
\hline
\end{tabular}


Balaji VCR et al. Int J Reprod Contracept Obstet Gynecol. 2021 Mar;10(3):1077-1088

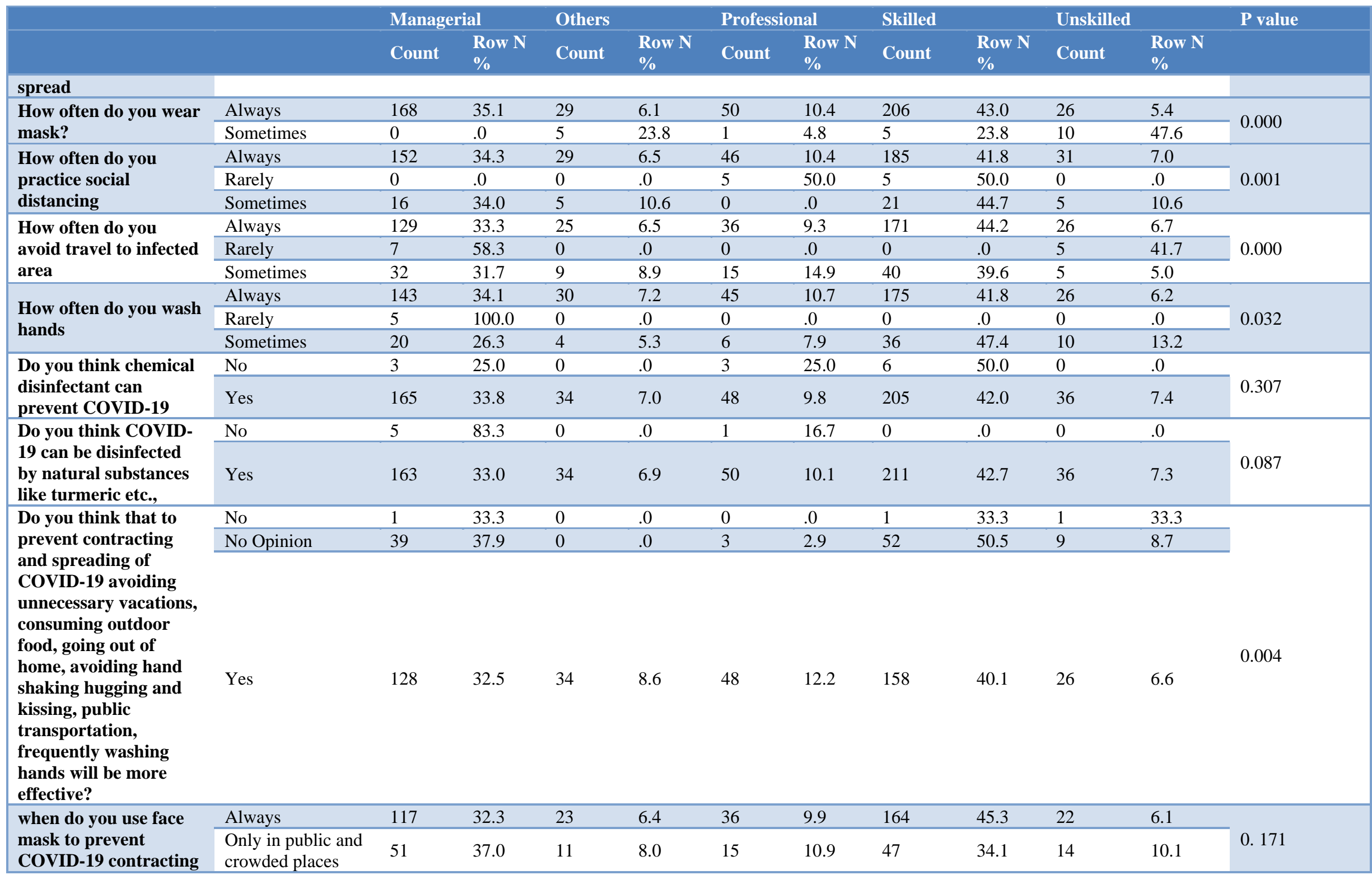


Balaji VCR et al. Int J Reprod Contracept Obstet Gynecol. 2021 Mar;10(3):1077-1088

Table 5: Study population ( $\mathrm{n=500)}$ compared by immediate family member suffering from COVID-19.

\begin{tabular}{|c|c|c|c|c|c|c|}
\hline & & \multicolumn{4}{|c|}{ Any Family Member Suffered from COVID-19 } & \multirow{3}{*}{ P value } \\
\hline & & \multicolumn{2}{|l|}{ No } & \multicolumn{2}{|l|}{ Yes } & \\
\hline & & Count & Row N \% & Count & Row N \% & \\
\hline $\begin{array}{l}\text { Avoid social distancing to prevent COVID- } \\
19 \text { spread }\end{array}$ & Yes & 434 & 86.80 & 66 & 13.20 & Not computable \\
\hline Wear mask to prevent spread of COVID-19 & Yes & 434 & 86.80 & 66 & 13.20 & Not computable \\
\hline \multirow{2}{*}{$\begin{array}{l}\text { Use hand sanitizer to prevent spread of } \\
\text { COVID-19 }\end{array}$} & No & 15 & 93.75 & 1 & 6.25 & \multirow{2}{*}{0.352} \\
\hline & Yes & 419 & 86.57 & 65 & 13.43 & \\
\hline \multirow{2}{*}{$\begin{array}{l}\text { Isolating people from infected area would } \\
\text { prevent COVID-19 spread }\end{array}$} & No & 5 & 100.00 & 0 & .00 & \multirow{2}{*}{0.491} \\
\hline & Yes & 429 & 86.67 & 66 & 13.33 & \\
\hline \multirow{2}{*}{ How often do you wear mask? } & Always & 418 & 87.27 & 61 & 12.73 & \multirow{2}{*}{0.129} \\
\hline & Sometimes & 16 & 76.19 & 5 & 23.81 & \\
\hline \multirow{3}{*}{ How often do you practice social distancing } & Always & 394 & 88.94 & 49 & 11.06 & \multirow{3}{*}{0.000} \\
\hline & Rarely & 10 & 100.00 & 0 & .00 & \\
\hline & Sometimes & 30 & 63.83 & 17 & 36.17 & \\
\hline \multirow{3}{*}{$\begin{array}{l}\text { How often do you avoid travel to infected } \\
\text { area }\end{array}$} & Always & 339 & 87.60 & 48 & 12.40 & \multirow{3}{*}{0.000} \\
\hline & Rarely & 5 & 41.67 & 7 & 58.33 & \\
\hline & Sometimes & 90 & 89.11 & 11 & 10.89 & \\
\hline \multirow{3}{*}{ How often do you wash hands } & Always & 363 & 86.63 & 56 & 13.37 & \multirow{3}{*}{0.680} \\
\hline & Rarely & 5 & 100.00 & 0 & .00 & \\
\hline & Sometimes & 66 & 86.84 & 10 & 13.16 & \\
\hline \multirow{2}{*}{$\begin{array}{l}\text { Do you think chemical disinfectant can } \\
\text { prevent COVID-19 }\end{array}$} & No & 12 & 100.00 & 0 & .00 & \multirow{2}{*}{0.179} \\
\hline & Yes & 422 & 86.48 & 66 & $13.52 \%$ & \\
\hline \multirow{2}{*}{$\begin{array}{l}\text { Do you think COVID-19 can be disinfected } \\
\text { by natural substances like turmeric etc., }\end{array}$} & No & 6 & 100.00 & 0 & .00 & \multirow{2}{*}{0.426} \\
\hline & Yes & 428 & 86.64 & 66 & 13.36 & \\
\hline \multirow{3}{*}{$\begin{array}{l}\text { Do you think that to prevent contracting and } \\
\text { spreading of COVID-19 avoiding } \\
\text { unnecessary vacations, consuming outdoor } \\
\text { food, avoiding going out of home, avoiding } \\
\text { hand shaking hugging and kissing, avoiding } \\
\text { public transportation, frequently washing } \\
\text { hands will be more effective? }\end{array}$} & No & 3 & 100.00 & 0 & .00 & \multirow{3}{*}{0.247} \\
\hline & $\begin{array}{l}\text { No } \\
\text { Opinion }\end{array}$ & 94 & 91.26 & 9 & 8.74 & \\
\hline & Yes & 337 & 85.53 & 57 & 14.47 & \\
\hline \multirow[b]{2}{*}{$\begin{array}{l}\text { when do you use face mask to prevent } \\
\text { COVID-19 contracting }\end{array}$} & Always & 315 & 87.02 & 47 & 12.98 & 0.460 \\
\hline & $\begin{array}{l}\text { Only in } \\
\text { public and } \\
\text { crowded } \\
\text { places }\end{array}$ & 119 & 86.23 & 19 & 13.77 & \\
\hline
\end{tabular}


Balaji VCR et al. Int J Reprod Contracept Obstet Gynecol. 2021 Mar;10(3):1077-1088

Table 6: Various study parameters by the level of education of the study population $(n=500)$.

\begin{tabular}{|c|c|c|c|c|c|c|c|c|c|c|c|c|}
\hline & & \multicolumn{2}{|c|}{ Collegiate } & \multicolumn{2}{|c|}{ Higher Secondary } & \multicolumn{2}{|l|}{ Middle } & \multicolumn{2}{|c|}{ No Formal } & \multicolumn{2}{|c|}{ Primary } & \multirow[b]{2}{*}{ P value } \\
\hline & & Count & $\begin{array}{l}\text { Row } \\
\text { N \% }\end{array}$ & Count & $\begin{array}{l}\text { Row } \\
\text { N \% }\end{array}$ & Count & $\begin{array}{l}\text { Row } \\
\text { N \% }\end{array}$ & Count & $\begin{array}{l}\text { Row } \\
\text { N \% }\end{array}$ & Count & $\begin{array}{l}\text { Row } \\
\text { N \% }\end{array}$ & \\
\hline $\begin{array}{l}\text { Avoid social } \\
\text { distancing to } \\
\text { prevent COVID- } \\
19 \text { spread }\end{array}$ & Yes & 120 & 24.00 & & 29.60 & 86 & 17.20 & 90 & 18.00 & 56 & 11.20 & $\begin{array}{l}\text { Not } \\
\text { computable }\end{array}$ \\
\hline $\begin{array}{l}\text { Wear mask to } \\
\text { prevent spread of } \\
\text { COVID-19 }\end{array}$ & Yes & 120 & 24.00 & 148 & 29.60 & 86 & 17.20 & 90 & 18.00 & 56 & 11.20 & $\begin{array}{l}\text { Not } \\
\text { computable }\end{array}$ \\
\hline \multirow{2}{*}{$\begin{array}{l}\text { Use hand sanitizer } \\
\text { to prevent spread } \\
\text { of COVID-19 }\end{array}$} & No & 1 & 6.25 & 0 & .00 & 5 & 31.25 & 10 & 62.50 & 0 & .00 & 0.000 \\
\hline & Yes & 119 & 24.59 & 148 & 30.58 & 81 & 16.74 & 80 & 16.53 & 56 & 11.57 & \\
\hline \multirow{2}{*}{$\begin{array}{l}\text { Isolating people } \\
\text { from infected area } \\
\text { would prevent } \\
\text { COVID-19 spread }\end{array}$} & No & 4 & 80.00 & 0 & .00 & 1 & 20.00 & 0 & .00 & 0 & .00 & 0.048 \\
\hline & Yes & 116 & 23.43 & 148 & 29.90 & 85 & 17.17 & 90 & 18.18 & 56 & 11.31 & \\
\hline \multirow{2}{*}{$\begin{array}{l}\text { How often do you } \\
\text { wear mask? }\end{array}$} & Always & 119 & 24.84 & 143 & 29.85 & 81 & 16.91 & 80 & 16.70 & 56 & 11.69 & 0.002 \\
\hline & Sometimes & 1 & 4.76 & 5 & 23.81 & 5 & 23.81 & 10 & 47.62 & 0 & .00 & \\
\hline \multirow{3}{*}{$\begin{array}{l}\text { How often do you } \\
\text { practice social } \\
\text { distancing }\end{array}$} & Always & 99 & 22.35 & 143 & 32.28 & 75 & 16.93 & 70 & 15.80 & 56 & 12.64 & 0.000 \\
\hline & Rarely & 5 & 50.00 & 5 & 50.00 & 0 & .00 & 0 & .00 & 0 & .00 & \\
\hline & Sometimes & 16 & 34.04 & 0 & .00 & 11 & 23.40 & 20 & 42.55 & 0 & .00 & \\
\hline \multirow{3}{*}{$\begin{array}{l}\text { How often do you } \\
\text { avoid travel to } \\
\text { infected area }\end{array}$} & Always & 76 & 19.64 & 133 & 34.37 & 70 & 18.09 & 66 & 17.05 & 42 & 10.85 & 0.000 \\
\hline & Rarely & 7 & 58.33 & 0 & .00 & 0 & .00 & 5 & 41.67 & 0 & .00 & \\
\hline & Sometimes & 37 & 36.63 & 15 & 14.85 & 16 & 15.84 & 19 & 18.81 & 14 & 13.86 & \\
\hline \multirow{3}{*}{$\begin{array}{l}\text { How often do you } \\
\text { wash hands }\end{array}$} & Always & 104 & 24.82 & 133 & 31.74 & 71 & 16.95 & 70 & 16.71 & 41 & 9.79 & 0.000 \\
\hline & Rarely & 0 & .00 & 5 & 100.00 & 0 & .00 & 0 & .00 & 0 & .00 & \\
\hline & Sometimes & 16 & 21.05 & 10 & 13.16 & 15 & 19.74 & 20 & 26.32 & 15 & 19.74 & \\
\hline \multirow{2}{*}{$\begin{array}{l}\text { Do you think } \\
\text { chemical } \\
\text { disinfectant can } \\
\text { prevent COVID- } \\
19\end{array}$} & No & 3 & 25.00 & 3 & 25.00 & 4 & 33.33 & 0 & .00 & 2 & 16.67 & 0.343 \\
\hline & Yes & 117 & 23.98 & 145 & 29.71 & 82 & 16.80 & 90 & 18.44 & 54 & 11.07 & \\
\hline \multirow[b]{2}{*}{$\begin{array}{l}\text { Do you think } \\
\text { COVID-19 can be } \\
\text { disinfected by } \\
\text { natural } \\
\text { substances like } \\
\text { turmeric etc., }\end{array}$} & No & 5 & 83.33 & 1 & 16.67 & 0 & .00 & 0 & .00 & 0 & .00 & 0.054 \\
\hline & Yes & 115 & 23.3 & 147 & 29.8 & 86 & 17.4 & 90 & 18.2 & 156 & 11.3 & Contil \\
\hline
\end{tabular}


Balaji VCR et al. Int J Reprod Contracept Obstet Gynecol. 2021 Mar;10(3):1077-1088

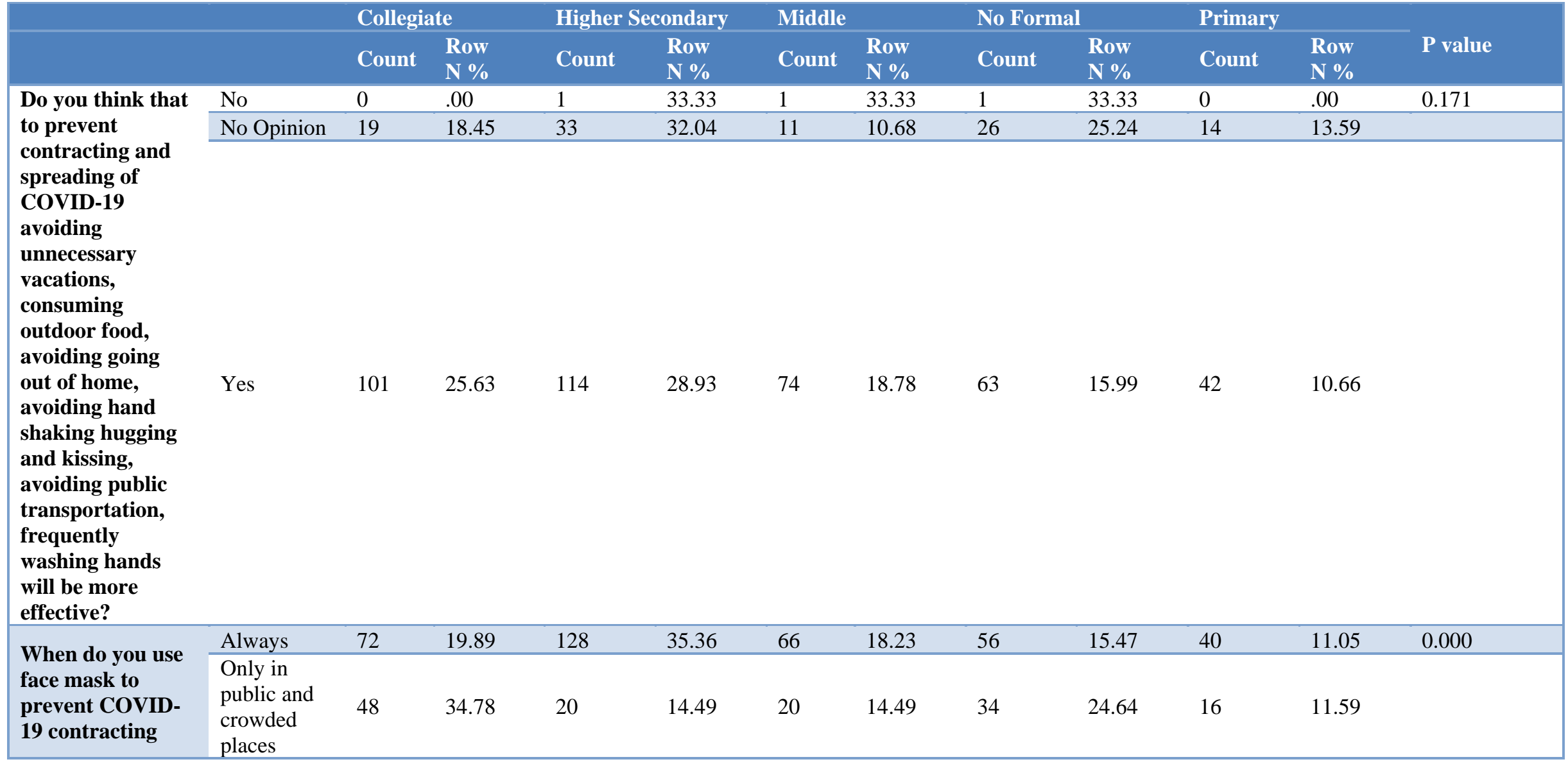




\section{DISCUSSION}

COVID-19 has spread worldwide and more than 10 million peoples are infected with death rate of approximately $6 \%$ which is higher than predicted $4 \%$ in beginning of disease. As per the last report, 74 cases were found per 1 million people in India. To control the same, government has implemented lockdown since March 25, 2020 well before any worst condition appears. After a long period of lockdown of various commercial activities, we need to understand the extent of public awareness towards COVID-19 pandemic so that an effective framework for creating awareness among public should be implemented keeping in view the existing public communication abilities including demographics, literacy levels, language spoken as well as socio-economic and cultural backgrounds. Various communities have been assessed highly vulnerable due to scarcity of natural resources and socio-cultural anomalies. ${ }^{4}$

A study carried out relating the swine influenza pandemic among the 1548 adults in Saudi Arabia concluded better level of awareness that will be reflected in higher tendency of behavioral precautions and thus can limit the spread of disease. Similarly, a study to investigate the awareness level among the adult population of Italy regarding avian influenza revealed poor level of knowledge-base and greater perceived risk due to poor precautionary behaviour among individuals and recommended health educational strategies. ${ }^{5,6}$

Scientific aptitude of survey participants is indicated by their answers related to structure, naming, origin, class of the virus, $48 \%$ of participants replied appropriately towards the scientific information, which indicates their ability to adopt innovative ways of various precautionary measures. Education, occupation and socio-economic conditions are found to be important in compliance of precautionary measures among study population in various studies related to $\mathrm{H} 5 \mathrm{~N} 1$ pandemics in various parts of the world. Self-organisation, communication and ability towards continuous learning. They serve as important tools for livelihood resilience, which will actually cope up with the shocks to reduce poverty, additionally will improvise upon community adaptive capacity building. ${ }^{?}$

Coronaviruses encompass a large family of viruses that can cause illnesses ranging from the common cold to more serious diseases such as severe acute respiratory syndrome (SARS) and Middle East respiratory syndrome. The virus that cause COVID-19 disease is named 2019$\mathrm{nCoV}$ virus which is a new species of coronavirus. All of the participants are aware of the virus. About $90 \%$ of participants were aware that washing hands frequently with soap and water for at least $20 \mathrm{~s}$, especially before eating, after using the bathroom, and after blowing your nose, coughing, or sneezing, and using hand sanitizer with at least $60 \%$ alcohol if soap and water are not available and wearing mask could prevent spread of
COVID-19. A cross-sectional survey on adults in US shows lacked critical knowledge about COVID-19 and, despite concern, were not changing routines or plans which results in most vulnerable communities. ${ }^{8}$

\section{CONCLUSION}

The study pointed out some important concern about the understanding of COVID-19 pandemic among South Indians. There is a clear need for training programme with respect to locale-specific scenario targeted to a specific cluster of population emplaning upon their respective lifestyle, to improve the knowledge and compliance about risk and preventions. Role of media, physician, government and non-governmental organizations and religious groups is extremely important in creating awareness about the various aspects of spread, prevention, treatment of the disease by means of interesting programmes, poems, songs, cartoons, talks, among others, to facilitate confidence of people to let them protect themselves, follow their economic activities and care COVID-19 patients.

Creating awareness by innovative ways should be adopted as one of the best practices to combat the spread of pandemic. Presentations on TV, social media in local people's friendly language, online and live competitions with continuous guidelines are required. There is a need to elaborate the Indian socio-cultural aspects so that society start appreciating and voluntarily following social distancing. This should improve the confidence of people to let them protect themselves not only from the present pandemic but also from all other unforeseen infections, provide care to patients, contribute towards country's economic build-up by maintaining livelihood resilience with continued presence and productivity at workplace. This should improve the confidence of people to let them protect themselves and care COVID-19 patients.s

\section{Limitations}

The significance of the findings of the study could have been strengthened greatly if the parameters of the study had been extended to include rural areas.

Funding: No funding sources

Conflict of interest: None declared

Ethical approval: The study was approved by the Institutional Ethics Committee

\section{REFERENCES}

1. Kakemam E, Nejad DG, Chegini Z, Momeni K, Salehinia H, Hassanipour S, Knowledge, attitudes, and practices among the general population during COVID-19 outbreak in Iran: a national crosssectional survey. Medrxiv. 2020;6:10-2.

2. Zaid AA, Barakat M, Rajaa A, Qudah A, Albetawi S, Hammad A. Knowledge and awareness of 
community toward COVID-19 in Jordan: A crosssectional study. Sys Rev Pharm. 2020;11(7):135-42.

3. Laxminarayan R, Wahl B, Dudala SR, Gopal K, Mohan BC. Epidemiology and transmission dynamics of COVID-19 in two Indian states. Science. 2020;370(6517):691-7.

4. Kaushik M, Agarwal D, Gupta AK. Cross-sectional study on the role of public awareness in preventing the spread of COVID-19 outbreak in India. Postgrad Med J. 2020;13:83-9.

5. Aly M, Khairy AA, Johani S, Balkhy H. Unusual rotavirus genotypes among children with acute diarrhea in Saudi Arabia. BMC Infect Dis. 2015;15:192.

6. Giuseppe G, Abbate R, Albano L, Marinelli P, Angelillo IF. A survey of knowledge, attitudes and practices towards avian influenza in an adult population of Italy. BMC Infect Dis. 2008;8:36.

7. Aly M, Khairy A, Johani S, Balkhy H. Unusual rotavirus genotypes among children with acute diarrhea in Saudi Arabia. BMC Infect Dis. 2015; 15:192.

8. Wolf MS, Serper M, Opsasnick L, O'Conor RM, Curtis L, Benavente JY. Awareness, attitudes, and actions related to covid-19 among adults with chronic conditions at the onset of the u.s. outbreak: a cross-sectional survey. Ann Intern Med. 2020;173(2):100-9.

Cite this article as: Balaji VCR, Nithya R.

Awareness regarding the strategies to bring down the pandemic among general population: a questionnaire based study. Int J Reprod Contracept Obstet Gynecol 2021;10:1077-88. 